\title{
Detecting price thresholds in choice models using a semi-parametric approach
}

\author{
Yasemin Boztuğ • Lutz Hildebrandt • \\ Kalyan Raman
}

(C) The Author(s) 2013. This article is published with open access at Springerlink.com

\begin{abstract}
The semi-parametric methodology, underutilized in marketing, can be applied to distinguish between competing models of price response and to estimate the model that most validly describes consumer response to price. The methodology is robust and flexible, thereby making it applicable to a wide spectrum of models of consumer response. In the specific context of reference prices, we show that the semi-parametric methodology helps the manager develop price promotions that most effectively capitalize on the nature of consumer price response.
\end{abstract}

Keywords Loss aversion · Threshold · Reference price · Semi-parametric estimation $\cdot$ Multinomial logit model

Financial support by the German Research Foundation (DFG) through the Sonderforschungsbereich 649, and for Boztug by way of research project \#B01952/1 is gratefully acknowledged.

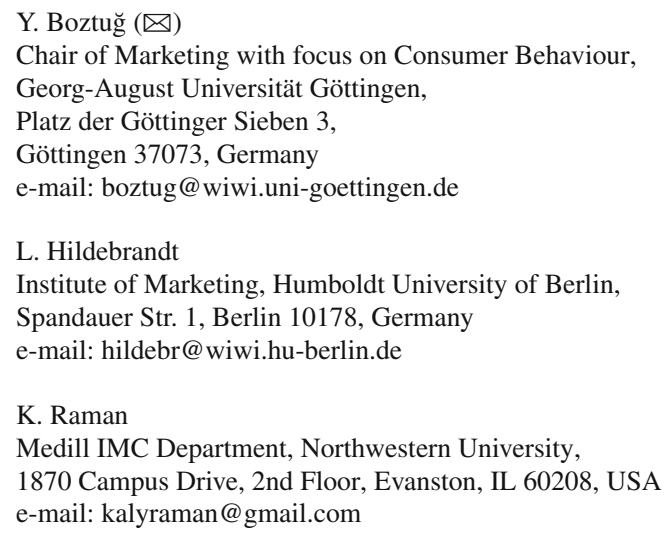




\section{Problem motivation and objective of our research}

Pricing decisions are a crucial determinant of consumer response and have considerable impact on the firm's profitability. Consequently, mistakes in setting or changing the price levels are costly to the firm. In particular, price promotions are important decisions that require careful structuring-for example, a price reduction may be achieved gradually or all at once, but these are two different ways of structuring the price reduction and they may elicit entirely different kinds of consumer response and different economic results. Thus, identification of the valid model for a particular market segment is critical because if we use an incorrect model, the price change may have no effect or-even worse-have exactly the opposite effect to that intended. Currently, there are two competing theories of consumer response to price with significantly different prescriptions for the implementation of price changes-one suggesting rapid changes in price and the other suggesting gradual changes in price depending upon whether a price increase or decrease is contemplated. At the heart of both the competing theories of consumer response to price is the notion of reference prices. Reference price theory postulates that consumers respond not only to the actual price but also to the deviation of the actual price from the reference price.

Several studies have shown that ignoring reference prices leads to incorrect conclusions about consumer behavior in purchase situations (e.g., Kalyanaram and Winer 1995). The two dominant theories of consumer response to price are built upon the deviation of the actual price from the reference price. Furthermore, consumer response may vary asymmetrically to positive and negative deviations. The two theories differ in how they treat these deviations. Range theory assumes that price variations within a latitude of acceptance are perceived to be less extreme than they are outside the latitude of acceptance. That is, within the latitude of acceptance, a price variation is perceived to be lower (higher) than it actually is. The existence of a latitude of acceptance is essentially equivalent to a non-zero threshold effect. Consumers respond more strongly to those deviations that exceed the threshold, as opposed to deviations below the threshold. On the other hand, the competing theory-closely related to prospect theory-assumes that any deviation of the actual price from the reference price, no matter how small it may be, has an effect on consumer response. Clearly, the latter theory assumes a zero threshold effect.

Kalyanaram and Winer (1995) urged distinguishing between these competing theories but, until now, this call has been answered in only limited fashion. Only infrequently do researchers test the two competing theories to identify the correct one- the danger here is that the wrong theory often gives significant results and a good fit to the data, thereby misleading researchers into drawing exactly the wrong conclusions about consumer response. Our contribution is to offer a non-parametric methodology that can identify the correct model and estimate consumer response to price more efficiently compared to existing techniques.

The rest of the paper is organized as follows: In the next section, we discuss the theoretical background for our research. We then describe our non-parametric and parametric methodology. Next, we present our empirical results using both simulation and real data. We conclude with a discussion of our results, managerial implications, limitations of this paper and suggestions for future research. 


\section{Theoretical background}

There is strong empirical evidence that consumer response is driven not solely by the price itself, but rather by its relation to the reference price of that product. Over the last three decades an enormous literature on this topic has been published, a large body of which is concerned with the subtleties involved in conceptualizing, defining and measuring reference prices (e.g., Biswas et al. 1993; Gijsbrechts 1993 ; Kalyanaram and Winer 1995; Mazumdar et al. 2005; Winer 1988).

The significance of the reference price concept for our research is straightforwardif the price exceeds the reference price, consumers perceive a loss, and if the price is below the reference price, consumers perceive a gain. The two competing theories of consumer response to changes in price differ on how they treat losses and gains. According to both schools of thought, consumers exhibit greater sensitivity to losses than to gains-a phenomenon known as loss-aversion. But one theory assumes that every price change is either a loss or gain regardless of how small the change might be (Kahneman and Tversky 1979; Tversky and Kahneman 1981). The other theory assumes that there is a latitude of indifference within which consumers are not sensitive to changes in price (Sherif and Hovland 1961; Janiszewski and Lichtenstein 1999; Niedrich et al. 2001). The latter theory is often called range theory — we will refer to it as loss aversion theory with a non-zero threshold (e.g., Kalyanaram and Little 1994; Han et al. 2001; Raman and Bass 2002; Pauwels et al. 2003). Our description distinguishes the latter theory from the former one in which there is no zone of indifference-we will refer to the former theory as loss aversion theory with zero threshold.

If loss aversion occurs with a non-zero threshold, the managerial implications are very different than if loss aversion occurs with zero threshold. A threshold effect is equivalent to a range of prices-a zone of indifference-within which people are indifferent to changes in price. In the absence of a threshold effect, there is no zone of indifference - therefore the price should not increase even in a gradual way but may be lowered in a gradual way. On the other hand, in the presence of a threshold effect, there is a zone of indifference within which price changes will be ineffectual-under these circumstances, price increases may be done gradually, but price reductions should not be made in a gradual way. This is because any price reduction within the zone of indifference will lower revenue without stimulating demand. Since price deviations require measurement of the reference price, we briefly discuss alternative methods reported in the literature for measuring reference prices. In Fig. 1, we give an overview how gains and losses influence the utility for a model of loss aversion with and without a threshold effect. Special emphasis should be given on the zone around zero [price equals the reference price $\left(P-P_{\text {ref }}=0\right)$ ], which shows how differently these two theories view consumer response to changes in price.

Because the reference price is a latent construct, it cannot be measured directly. One way of measuring it is to conduct experiments or to ask consumers to state their expected price but each of these methods have their own problems, as discussed in Estelami et al. (2001). Scanner data provide other information that can be used to estimate a reference price. There are two ways of accomplishing this. One method uses prices paid in the past and the other uses current prices. In the first approach, it is assumed that consumers use prices paid in the past to construct their reference price; 


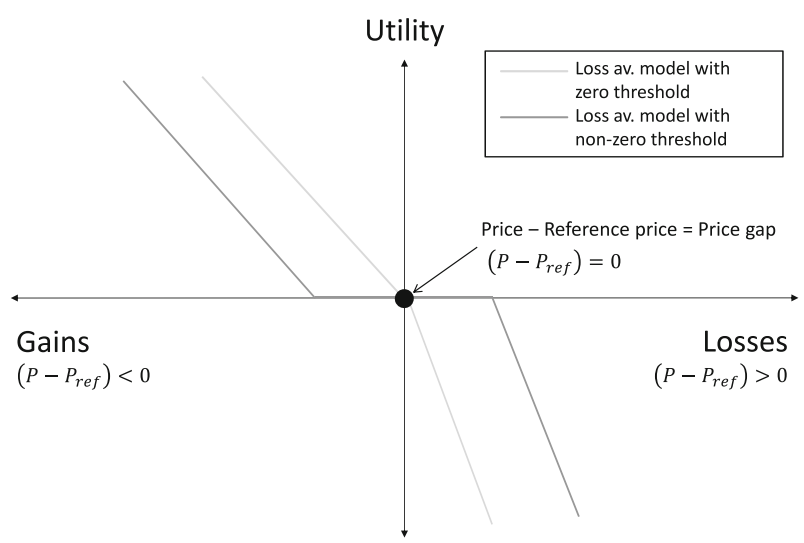

Fig. 1 General figure for loss aversion model with zero threshold and loss aversion model with non-zero threshold

this is called an "internal reference price" (e.g., Winer 1986). In the second approach, it is assumed that consumers use prices at the point of sale, which are actual prices; this is called an "external reference price" (e.g., Hardie et al. 1993). For an empirical comparison of both methods, see, for example, Briesch et al. (1997).

Clearly, the internal reference price applies only to consumers who are able to recall past prices. These consumers are likely to be either highly involved shoppers or price-sensitive consumers (e.g., Rajendran and Tellis 1994). They have an internal reference price for each brand. There are various formulations for computation of an internal reference price, but none of them have become standard practice (e.g., Wricke et al. 2000).

The external reference price is based on the current prices at the point of purchase. It is not brand-specific, but only category-specific. It is assumed that the consumer does not use any information on past purchases, perhaps because he cannot remember past prices paid or does not trust his memory of them. Therefore, this type of consumer uses only information that is available during the current shopping trip. As with the internal reference price, the literature provides no consensus about the best way to measure external reference price.

\section{Description of the methodology}

\subsection{General discussion on parametric and non-parametric estimation methods}

Researchers have traditionally relied heavily upon parametric estimation methods. We supplement the traditional methodology with a sophisticated non-parametric technique to identify the correct theory rather than impose one theory or the other upon the data as parametric techniques do. Non-parametric techniques do not commit themselves to specific functional forms to describe the input-output relationship. Consequently, they are flexible and very well suited to let "the data speak for themselves." However, non-parametric techniques are not the same as 'data mining' which is devoid of theory; 
in fact non-parametric techniques can identify from competing theories that one which best describes the data.

Our methodology is best described as semi-parametric because we combine both non-parametric and parametric approaches. The semi-parametric approach has a number of attractive features. For example, when the correct theory turns out to be loss aversion with a threshold, the methodology automatically yields estimates of the threshold points. Another advantage of the semi-parametric technique is that it does not assume symmetric threshold effects as parametric methods do. Finally, the semi-parametric method offers a more efficient approach to dealing with consumer heterogeneity issues. For example, one source of heterogeneity is that some consumers are brand loyal and others are not. Suppose the market is segmented by loyalty. Then within each segment-loyal and non-loyal—-there are two possible models: one with and another without a threshold effect. Consequently, using parametric techniques, we would have to estimate four possible models. The semi-parametric method could potentially reduce the estimation complexity because if it identifies the correct model to be loss aversion with no threshold, then we need not deal with the estimation of threshold effects, which is typically a complex matter.

While the non-parametric approach is powerful precisely because it assumes no specific functional forms, that very strength is also a weakness in that the non-parametric model has no controllable parameters that the manager can use to fine-tune the marketing program. However, this issue may be addressed in the following manner. We can compute the slopes of the utility function at all the price points in the gain regime, repeat the procedure in the loss regime, and then determine the average slope. This can then be used as the imputed beta in the model to find optimal prices and promotions in the three regimes under consideration-gain, loss, and indifference. Admittedly, this is a purely numerical recipe and lacks the elegance of an analytical closed-form result. While the lack of analytical optimality results may seem dissatisfying from an academic point of view, we get an easy implementable pricing algorithm that adds value from a managerial perspective.

We propose the following two-stage procedure. First, estimate a reference price model with a non-parametric price response function without pre-supposing either loss aversion with zero threshold or loss aversion with non-zero threshold. One of the results of the non-parametric estimation is the discovery of threshold levels. Second, given the identified theory from the non-parametric phase, estimate a parametric model representing that theory, and, if necessary, use the non-parametrically determined width of the indifference zone for the model. Note that the model of loss aversion with a threshold subsumes the model of loss aversion without a threshold. If no zone of indifference exists, then the model of loss aversion with a threshold will estimate the threshold to be zero. The semi-parametric approach facilitates detection of the threshold-it combines identification of the true model and its estimation in one fell swoop.

\subsection{Description of algorithms}

Our algorithm uses individual level data. The general model structure is an additive random utility framework (e.g., McFadden 1974), as described below, where $U_{i n}$ is the overall utility of brand $i$ for consumer $n, V_{i n}$ is his systematic utility component 
and $\varepsilon_{i n}$ is the random component.

$$
U_{i n}=\underbrace{V_{i n}}_{\text {systematic utility }}+\underbrace{\varepsilon_{i n}}_{\text {random component }} .
$$

Different formulations - either parametric or non-parametric-can be specified for the systematic and random components. In one of our formulations, both components are described in a parametric way and the model can be formulated as a multinomial logit model (e.g., McFadden 1974). The other model is a semi-parametric one resulting from a non-parametric formulation of the systematic part and parametric modeling of the random component. A suitable model description for this type is a generalized additive model (e.g., Hastie and Tibshirani 1990).

\subsubsection{Parametric approach}

A well-known disaggregated choice model is the multinomial logit model (MNL), which was introduced in the marketing context by Guadagni and Little (1983). Here, both the systematic component and the random component are modeled parametrically. For the difference of the errors, we assume a Gumbel distribution as is standard practice in the literature on MNL models. The model structure is linear-additive. A popular MNL model is:

$$
\operatorname{Pr}_{n}(i)=\frac{\exp \left(\beta^{\prime} x_{i n}\right)}{\sum_{j \in C_{n}} \exp \left(\beta^{\prime} x_{j n}\right)}
$$

where $\operatorname{Pr}_{n}(i)$ is the probability of individual $n$ to buy product $i, x_{i n}$ the explanatory variables of product $i$ for consumer $n$, and $\beta$ the parameter vector to be estimated. We use maximum-likelihood estimation.

\subsubsection{Semi-parametric approach}

Our semi-parametric method provides an alternative to the MNL model. Here, we focus on a generalized additive model (GAM) (Hastie and Tibshirani 1990). ${ }^{1}$ This method has several advantages. The error structure can be modeled as in the MNL framework, thus facilitating comparison of the estimation results. The systematic component of a GAM has an additive structure, as does the MNL. The main difference from the MNL is a one-dimensional non-parametric function for each explanatory variable, in contrast to the linear modeling of the explanatory variables in a MNL. Consider the conditional expectation of $Y$, given a specific value $x$ of $X$, denoted by the notation $E[Y \mid X=x]$. We use conditional expectations, because this is the standard way to describe models in a generalized way (also see the description for generalized linear models (McCullagh and Nelder 1989)). The formal model of a GAM is presented in

\footnotetext{
1 An alternative approach using P-Splines is used by Steiner et al. (2007), but their main focus is on estimating price response functions rather than incorporating any reference price.
} 
Eq. (2).

$$
E[Y \mid X=x]=G\left(\sum_{p} f_{p}\left(x_{p}\right)\right) \quad \text { with } \quad G=\frac{1}{1+\exp (-x)}
$$

Here, the $f_{p}$ are one-dimensional non-parametric functions to be estimated and $x_{p}$ are the explanatory variables; $G$ is called a link function. By specifying $G$ as in Eq. (2), the error term distribution of the GAM is the same as in the MNL approach.

The standard GAM is defined only for continuous explanatory variables. In marketing applications, there are usually also categorical explanatory variables, for example, display or feature. To incorporate these variables, the common GAM is extended to include a linear-additive component, as in the MNL model. Our extended GAM is defined in Eq. (3).

$$
E[Y \mid X=x]=G\left(\sum_{p} f_{p}\left(x_{p}\right)+\beta^{\prime} x\right)
$$

In Eq. (3), $\beta^{\prime} x$ is the linear component, and $x$ contains both continuous and categorical variables. Two different algorithms exist for estimating a GAM. The most common approach is backfitting, introduced by Friedman and Stuetzle (1981). The method is based on a variance decomposition of the total variance into the variance components accounted for all explanatory variables specified in the model. An alternative method is called marginal integration, where the marginal influences of the explanatory variables to the response variable are estimated. We do not describe the mathematical structure of the estimation procedures in detail because the reader will find excellent treatments of the topic in Hastie and Tibshirani (1990) or Linton and Nielsen (1995).

\subsubsection{Integration of the reference price concept in a parametric model}

The integration of the reference price into the standard choice model is achieved by defining two components which describe the loss or gain perceived by the consumer. The pure price component should be included only in a model with an internal reference price formulation. In using an external reference price, the price component has to be excluded due to strong correlations with the gain and/or loss components (e.g., Hardie et al. 1993). The resulting model describing the utility $U_{i n t}$ of consumer $i$ for product $n$ at time $t$ is shown in Eq. (4).

$$
\begin{aligned}
U_{i n t}= & \beta_{0}+\beta_{1} P_{i n t}+\beta_{2} \underbrace{\left(P_{i n t}-I R P_{i n t}\right) I_{P_{i n t}>I R P_{i n t}}}_{\text {Loss }} \\
& +\beta_{3} \underbrace{\left(I R P_{i n t}-P_{i n t}\right) I_{I R P_{i n t}}>P_{i n t}}_{\text {Gain }} \\
& +\beta_{4}{ }_{L O Y_{i n t}}+\beta_{5} D_{I S P L_{i n t}}+\beta_{6} \text { FEAT }_{i n t}+\varepsilon_{i n t}
\end{aligned}
$$


In Eq. (4) IRP is the internal reference price. The variable ' $I$ ' is an indicator variable which takes on the value when the condition indicated in its subscript is satisfied and is zero otherwise. For example, the variable $I_{P_{i n t}>I R P_{i n t}}$ is equal to 1 when $P_{\text {int }}>I R P_{\text {int }}$ - thus is equal to 1 only when the price exceeds the internal reference price. Consequently, the price deviation $\left(P_{i n t}-I R P_{i n t}\right)$ will influence the utility only when the price exceeds the internal reference price. The corresponding parameter $\beta_{2}$ measures the sensitivity of the utility to increases in price above the internal reference price. The gain and loss components come into play only when the internal reference price is larger or smaller, respectively, than the actual price. All other explanatory variables are included as in a standard logit model, for example, $P$ for price, $L O Y$ for loyalty, as specified by Guadagni and Little (1983), DISPL for the binary variable for the existence of display, and, in the same manner, FEAT for feature. The parameter vector $\beta$ contains the parameters to be estimated.

In a loss aversion model with non-zero threshold, a mid range, also known as a range of indifference, must be included. Here, we assume again that we have loss and gain components (Kalyanaram and Little 1994). ${ }^{2}$ In the formal representation of a loss aversion model with non-zero threshold (Eq. (5)), in addition to the common variables, as specified for Eq. (4), two additional parameters, $\delta_{\text {Gain }}$ and $\delta_{\text {Loss }}$, need to be specified. The sum of $\delta_{\text {Gain }}$ and $\delta_{\text {Loss }}$ describes the width of the range of indifference. In the model presented here, we assume a non-symmetric indifference zone around the price gap (price minus reference price). This is a generalization of the model presented by Kalyanaram and Little (1994).

$$
\begin{aligned}
& U_{i n t}=\beta_{0}+\beta_{1} P_{i n t}+\beta_{2} \underbrace{\left(P_{i n t}-I R P_{i n t}\right) I_{P_{i n t}>I R P_{i n t}+\delta_{\text {Loss }}}}_{\text {Loss }} \\
& +\beta_{3} \underbrace{\left(P_{\text {int }}-I R P_{\text {int }}\right) I_{\text {IRP }} \text { int }-\delta_{\text {Gain }}<P_{\text {int }}<I R P_{\text {int }}+\delta_{\text {Loss }}}_{\text {Mid }} \\
& +\beta_{4} \underbrace{\left(I R P_{i n t}-P_{i n t}\right) I_{P_{\text {int }}<I R P_{\text {int }}-\delta_{\text {Gain }}}}_{\text {Gain }} \\
& +\beta_{5} L O Y_{i n t}+\beta_{6} D I S P L_{i n t}+\beta_{7} F E A T_{i n t}+\varepsilon_{i n t}
\end{aligned}
$$

In a parametric approach, it is nearly impossible to detect $\delta_{\text {Gain }}$ and $\delta_{\text {Loss }}$ if these are assumed to be unequal. Even in the symmetric case, a grid search for $\delta_{\text {Gain }}$ and $\delta_{\text {Loss }}$ is laborious. This is where the non-parametric approach enjoys a decisive advantage-it facilitates much easier estimation of unequal $\delta_{\text {Gain }}$ and $\delta_{\text {Loss }}$.

\footnotetext{
2 In alternative approaches, Han et al. (2001), Raman and Bass (2002), and Pauwels et al. (2003) use models with price threshold components. The price threshold models are not clearly based on assimilation contrast theory; they seem to be more data driven (Raman and Bass present some theory, but their model is at the aggregate level and they use only a reference price and not the difference between price and the reference price).
} 


\subsubsection{Integration of the reference price concept in a semi-parametric approach}

The semi-parametric estimation combines non-parametric and parametric terms in the specification of the utility function. All continuous explanatory variables can be modeled with a one-dimensional non-parametric function. In a reference price context, these variables are loyalty, price gap, and price. Price gap is defined as price minus reference price. It is negative for a gain and positive for a loss. Equation (6) does not include a non-parametric function for price because the price component is used only in models with an internal reference price effect (see the section on loss aversion above).

$$
\begin{aligned}
U_{i n t}= & \beta_{0}+\beta_{1} P_{i n t}+f_{1}\left(P R I C E G A P_{i n t}\right)+f_{2}\left(L O Y_{i n t}\right) \\
& +\beta_{2} D_{I S P L_{i n t}}+\beta_{3} F E A T+\varepsilon_{i n t}
\end{aligned}
$$

The $f_{i}(i=1,2)$ is a one-dimensional non-parametric function [see Eq. (2) for more explanation]. In many models, using a non-parametric function for loyalty leads to an approximately linear functional form (see e.g., van Heerde et al. 2001). The key non-parametric component is the price gap. Therefore, our semi-parametric approach reduces to an equation with only one non-parametric function for the price gap. By inspecting the estimated functional form of the price gap, the researcher can determine whether loss aversion with zero or non-zero threshold provides the best description for the data. ${ }^{3}$

\section{Empirical application}

\subsection{Simulation study}

We conducted a set of simulations with two goals in mind. First, we wanted to show the consequences of estimating a model representing one theory, for example, a loss aversion model with non-zero threshold, when the data in fact follow the other theorynamely, a loss aversion model with zero threshold. Second, we wanted to illustrate the power of the semi-parametric approach through its ability to detect the correct theory underlying the data.

We generated data sets based on a sample size of 5,000 purchases for 200 households - the consumer choices in one data set reflected loss aversion with a nonzero threshold, and the consumer choices in the other data set reflected loss aversion with a zero threshold. This leads to four possible scenarios corresponding to estimating each of the two possible models using data generated by the same or by the other model. Two additional scenarios correspond to estimating the semi-parametric model on each of the two data sets-if the semi-parametric method works, it should correctly identify the non-zero (zero) threshold for the data set that was generated according to a loss aversion model with non-zero (zero) threshold. In Table 2, we give an overview showing which tables and figures display our estimation results. Thus, we have esti-

\footnotetext{
3 For example, Pauwels et al. (2003) remark that fully non-parametric models have high data requirements, which is not the case in our approach because we model only one component non-parametrically.
} 
Table 1 Variable specification for simulation study

\begin{tabular}{llll}
\hline & Brand 1 & Brand 2 & Brand 3 \\
\hline Price & Uniform [0.75; 1.00] & Uniform [0.65; 0.90] & Uniform [0.60; 0.70] \\
Promoted price & Uniform [0.50;0.70] & Uniform [0.40; 0.55] & Uniform [0.45; 0.55] \\
$\begin{array}{l}\text { Promotional } \\
\text { frequency }\end{array}$ & $\begin{array}{c}\text { Randomly 1 out of } 4 \\
\text { weeks }\end{array}$ & $\begin{array}{c}\text { Randomly 1 out of 4 } \\
\text { Feeks }\end{array}$ & $\begin{array}{c}\text { Randomly 1 out of 8 } \\
\text { weeks }\end{array}$ \\
& $\begin{array}{c}\text { Randomly 1 out of 16 frequency } \\
\text { weeks }\end{array}$ & $\begin{array}{c}\text { Randomly 1 out of 16 } \\
\text { weeks }\end{array}$ & $\begin{array}{c}\text { Randomly 1 out of 36 } \\
\text { weeks }\end{array}$ \\
\hline
\end{tabular}

Table 2 Description of which table displays the estimation results for each of the six scenarios corresponding to three estimation methods used on two data sets

\begin{tabular}{|c|c|c|}
\hline \multirow[t]{2}{*}{ Estimation approach } & \multicolumn{2}{|l|}{ Data generating approach } \\
\hline & $\begin{array}{l}\text { Loss aversion model with zero } \\
\text { threshold }\end{array}$ & $\begin{array}{l}\text { Loss aversion model with non-zero } \\
\text { threshold }\end{array}$ \\
\hline $\begin{array}{l}\text { Loss aversion model with } \\
\text { zero threshold }\end{array}$ & Table 3,2 nd column and Fig. $2 \mathrm{a}$ & Table 4, 4th column and Fig. 3a \\
\hline $\begin{array}{l}\text { Loss aversion model with } \\
\text { non-zero threshold }\end{array}$ & Table 3, 3rd column and Fig. 2b & $\begin{array}{l}\text { Table 4, 2nd (assumption of } \\
\text { asymmetric threshold) and 3rd } \\
\text { (assumption of symmetric } \\
\text { threshold) column and Fig. 3b }\end{array}$ \\
\hline Semi-parametric approach & Table 3 , 4th column and Fig. 2c & Table 4, 5th column and Fig. 3c \\
\hline
\end{tabular}

mation results for a total of six possible scenarios and these are reported in Tables 3 and 4 . The data sets contain three brands and three explanatory variables-price, promotional price, and feature. The parameter values for the $\beta$ s are chosen as can be seen in Table 3, respectively, in Table 4 in the column named "true model".

The research design is comparable to that of Chang et al. (1999); however, in our study, we included an internal reference price and used a loss aversion model with zero threshold to generate one data set, and a loss aversion model with non-zero threshold to generate the other data set. The error term was created based on the standard MNL assumption. Thus, we calculated the utility of each brand for each observation based on the model with the underlying theory. Following random utility theory, the brand with the highest utility was "chosen" by the consumers. Variable specifications are laid out in Table 1. In the interest of improving the clarity of the tables with the estimation results (Tables 3, 4, 5, 6) - which already contain a lot of information-we report only the key results. However, the first author will be happy to supply the rest of the empirical results available to interested readers.

For generating the data set based on a loss aversion model with non-zero threshold, we assumed an asymmetric range of indifference with $\delta_{\text {Gain }}=0.2$ and $\delta_{\text {Loss }}=0.1$. This asymmetric assumption follows from Kalyanaram and Little (1994), who proposed that an asymmetric range is much more plausible than a symmetric one. According to a loss aversion model with zero threshold, consumers will react more strongly to a loss than to a gain, which leads to the assumption that the indifference zone should be smaller on the loss side than it is on the gain side. 
Table 3 Estimation results for simulated data set based on a loss aversion model with zero threshold

\begin{tabular}{lllll}
\hline & True model & $\begin{array}{l}\text { Loss aversion model } \\
\text { with zero threshold }\end{array}$ & $\begin{array}{l}\text { Loss aversion model } \\
\text { with non-zero threshold }\end{array}$ & $\begin{array}{l}\text { Semi-parametric } \\
\text { approach }\end{array}$ \\
\hline$P$ & -7 & $-\mathbf{7 . 4 2}^{\mathrm{a}}$ & $-\mathbf{7 . 4 2}^{\mathrm{a}}$ & $-\mathbf{7 . 4 0}^{\mathrm{a}}$ \\
FEAT & 2 & $\mathbf{2 . 1 6}^{\mathrm{a}}$ & $\mathbf{2 . 1 6}^{\mathrm{a}}$ & $\mathbf{2 . 1 4}^{\mathrm{a}}$ \\
Gain & 4 & $\mathbf{4 . 3 3}^{\mathrm{a}}$ & $\mathbf{4 . 3 3}^{\mathrm{a}}$ & Plot \\
Loss & -6.5 & $-\mathbf{6 . 2 8}^{\mathrm{a}}$ & $-\mathbf{6 . 2 8}^{\mathrm{a}}$ & Plot \\
$\delta$ & 0 & n. a. & 0 & $-2,262$ \\
$\ell$ & & $-2,263$ & $-2,263$ & 0.495 \\
$\bar{\rho}^{2}$ & & 0.495 & 0.495 & \\
\hline
\end{tabular}

a True parameter value lies within the $95 \%$ confidence interval. Bolded values indicate significant parameter estimates

Table 4 Estimation results for simulated data set based on a loss aversion model with non-zero threshold

\begin{tabular}{llllll}
\hline & $\begin{array}{l}\text { True } \\
\text { model }\end{array}$ & $\begin{array}{l}\text { Loss aversion model } \\
\text { with non-zero threshold, } \\
\delta \text { Asymmetric }\end{array}$ & $\begin{array}{l}\text { Loss aversion model } \\
\text { with non-zero threshold, } \\
\delta \text { symmetric }\end{array}$ & $\begin{array}{l}\text { Loss aversion } \\
\text { model with } \\
\text { zero threshold }\end{array}$ & $\begin{array}{l}\text { Semi- } \\
\text { parametric } \\
\text { approach }\end{array}$ \\
\hline$P$ & -7 & $\mathbf{- 7 . 4 2}^{\mathrm{a}}$ & $-\mathbf{7 . 8 5}^{\mathrm{a}}$ & $-\mathbf{7 . 6 0}^{\mathrm{a}}$ & $\mathbf{- 7 . 2 6}^{\mathrm{a}}$ \\
FEAT & 2 & $\mathbf{2 . 0 2}^{\mathrm{a}}$ & $\mathbf{1 . 8 7}^{\mathrm{a}}$ & $\mathbf{1 . 8 3}^{\mathrm{a}}$ & $\mathbf{2 . 0 3}^{\mathrm{a}}$ \\
Gain & 4 & $\mathbf{3 . 9 2}^{\mathrm{a}}$ & $\mathbf{3 . 2 1}^{\mathrm{a}}$ & $\mathbf{2 . 9 6}$ & \\
Mid & 0.05 & 0.52 & -0.68 & n. a. & Plot \\
Loss & -6.5 & $\mathbf{- 6 . 2 4}$ & $\mathbf{- 5 . 9 8}$ & $\mathbf{- 5 . 3 0}$ & \\
$\delta$ & $0.2 / 0.1$ & $0.2 / 0.1$ & $0.1 / 0.1$ & n. a. & Plot \\
$\ell$ & & $-2,383$ & $-2,409$ & $-2,434$ & $-2,386$ \\
$\bar{\rho}^{2}$ & 0.469 & 0.463 & 0.458 & 0.468 \\
\hline
\end{tabular}

a True parameter value lies within the $95 \%$ confidence interval. Bolded values indicate significant parameter estimates

\subsubsection{Data generated by loss aversion with zero threshold}

The first simulation study describes the results for the data set generated from a loss aversion model with zero threshold. In Table 3 and Fig. 2, we present the parametric and semi-parametric results, respectively.

In Table 3, we show the main estimation results for the simulated data set based on a loss aversion model with zero threshold. In the table, we present in the first column the true values for the $\beta \mathrm{s}$ and the size of the indifference zone as denoted of the generated data set. In the second column, we present the estimation results for a model which follows a loss aversion model with zero threshold. It can be shown that when estimating a model for loss aversion with zero threshold using the data set based on a loss aversion model with zero threshold, all true parameter values lie within the approximate $95 \%$ confidence interval and are significant.

When estimating a loss aversion model with non-zero threshold on this data set, as presented in column three, the grid search for $\delta_{\text {Gain }}+\delta_{\text {Loss }}$ leads to an "optimal" 

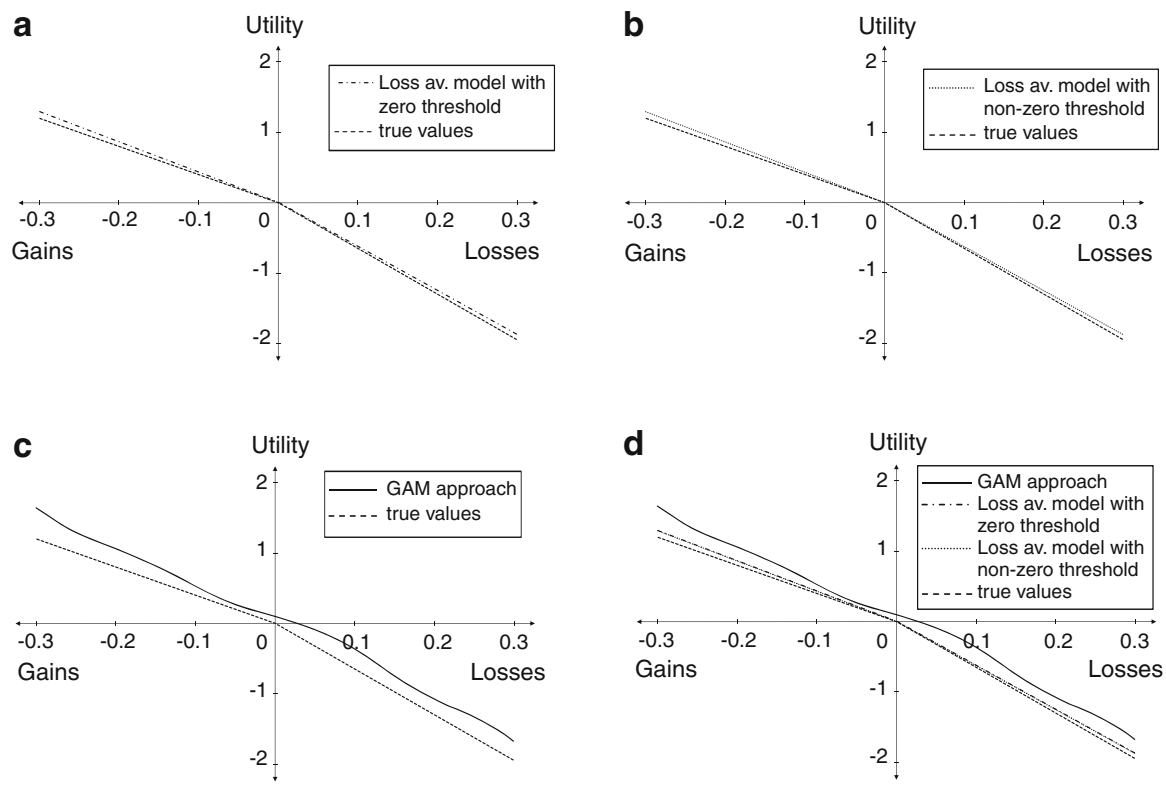

Fig. 2 Semi-parametric and parametric estimation for the price gap in the simulation study with data based on a loss aversion model with zero threshold. a The estimation for a model based on loss aversion with zero threshold. b The estimation for a model based on loss aversion with non-zero threshold. $\mathbf{c}$ The semi-parametric estimation, and $\mathbf{d}$ a combination of $\mathbf{a}-\mathbf{c}$

value of 0 , so the model reduces to one regarding a loss aversion model with zero threshold. Therefore, all estimated values for $\beta$ are equal to those based on the loss aversion model with zero threshold (as shown in columns 2 and 3, respectively).

For the semi-parametric model, we specify price and feature in a parametric manner, and the price gap (price minus reference price) in a non-parametric way. The results for the non-parametric estimation are given in column 4 and they are displayed graphically in Fig. 2c, d. The horizontal axis in Fig. 2 represents the price gap and the vertical axis denotes the effect of the price gap on the utility function (see Fig. 1 for a general picture as well). The parameter estimates are all significant and the approximate $95 \%$ confidence interval includes the true parameter values. The semi-parametric model correctly identifies the model that generated these data. This results hold for the parametrically estimated sss for price and feature, as well as for the non-parametric estimation of the price gap as presented in Fig. 2. In addition, we give the values for the log-likelihood value $(l)$ and the corrected likelihood ratio index $\left(\bar{\rho}^{2}\right)$, which accounts for the number of additional explanatory variables.

\subsubsection{Data generated by a loss aversion model with non-zero threshold}

In the second simulation study, the results for the data set generated from a loss aversion model with non-zero threshold are described. The indifference zone for the simulated data is asymmetric. We estimate two different models based on a loss aversion model with non-zero threshold: one with the true asymmetric values for $\delta_{\text {Gain }}$ and $\delta_{\text {Loss }}$ and 
one with symmetric $\delta_{\text {Gain }}$ and $\delta_{\text {Loss }}$ values, as suggested by Kalyanaram and Little (1994). All parametric estimation results for a loss aversion model with non-zero threshold are presented in columns 2 and 3 in Table 4.

With a non-zero threshold, the slope of the utility function is zero in the mid-range (the latitude of indifference). Consequently, we would expect the parameter estimates to be insignificant in the mid-range. This result can be found in columns 2 and 3 at the "Mid" row. As you can see, for both approaches the parameters are insignificant. All other parameter estimates are significant (for price, feature, gain and loss), and all the approximate confidence intervals include the true parameter values. In the model with the true $\delta_{\text {Gain }}$ and $\delta_{\text {Loss }}$ values, the estimated parameters are closer to the real values than the estimates obtained using the symmetric $\delta_{\text {Gain }}$ and $\delta_{\text {Loss }}$ values; the $\log$ likelihood is also better in this model than in the one with symmetric values for $\delta_{\text {Gain }}$ and $\delta_{\text {Loss }}$. In the model with a symmetric indifference zone, we find an optimal width of $\delta_{\text {Gain }}=\delta_{\text {Loss }}=0.1$, so that, $\delta_{\text {Gain }}+\delta_{\text {Loss }}=0.2$, which is smaller than the original value. But using an indifference zone than its true value still leads to much better estimation results than using a wrong approach, as we will demonstrate next.

Using the wrong model for this data set corresponds to estimating a loss aversion model with zero threshold. Doing so yields the following findings, which are presented in Table 4 in column 4 . First, every parameter estimate is still significant. Second, the approximate confidence intervals of loss and gain do not include the true parameter values, and this is something that would be undiscovered with real data. Although the estimates are significant, they are actually not even close to the true underlying parameters. Therefore, parametric estimation with the wrong model may still generate significant parameters - despite misspecification, thereby misleading the researcher.

We now turn to the results of the semi-parametric approach. Again, both parametrically estimated parameters are significant and their approximate confidence intervals include the true values. The non-parametric estimates are shown in Fig. 3c, d.

The functional form of the non-parametric part of the semi-parametric model is close to the real values, as is the asymmetric indifference zone; clearly, a loss aversion model with non-zero threshold is at work. Figure $3 \mathrm{c}, \mathrm{d}$ illustrates the boundaries of the indifference zone. From the figure, it is obvious that the estimates of a loss aversion model with zero threshold are wrong, especially in the zone around the price gap, which is the most relevant area for price discounts.

Several conclusions follow from the simulation study results. Estimating the correct model for the data yields significant parameter estimates whose approximate $95 \%$ confidence intervals include the true parameters. Using the "wrong" model for the data can produce three different outcomes. First, and most important, using a loss aversion model with zero threshold on a data set that came from a loss aversion model with nonzero threshold leads to significant parameter estimates, but the approximate confidence interval will not include the true parameters. This fact would remain undiscovered for a real data set. Second, if a loss aversion model with zero threshold is the correct description of the data, estimating the "wrong" model—a loss aversion model with non-zero threshold - will still produce correct results because the estimated "optimal" width for the indifference zone will be zero. Third, regardless of which theory is the "correct" one for a data set, the semi-parametric estimates will clearly indicate which model should be used. If it turns out that the semi-parametric estimates recommend the 

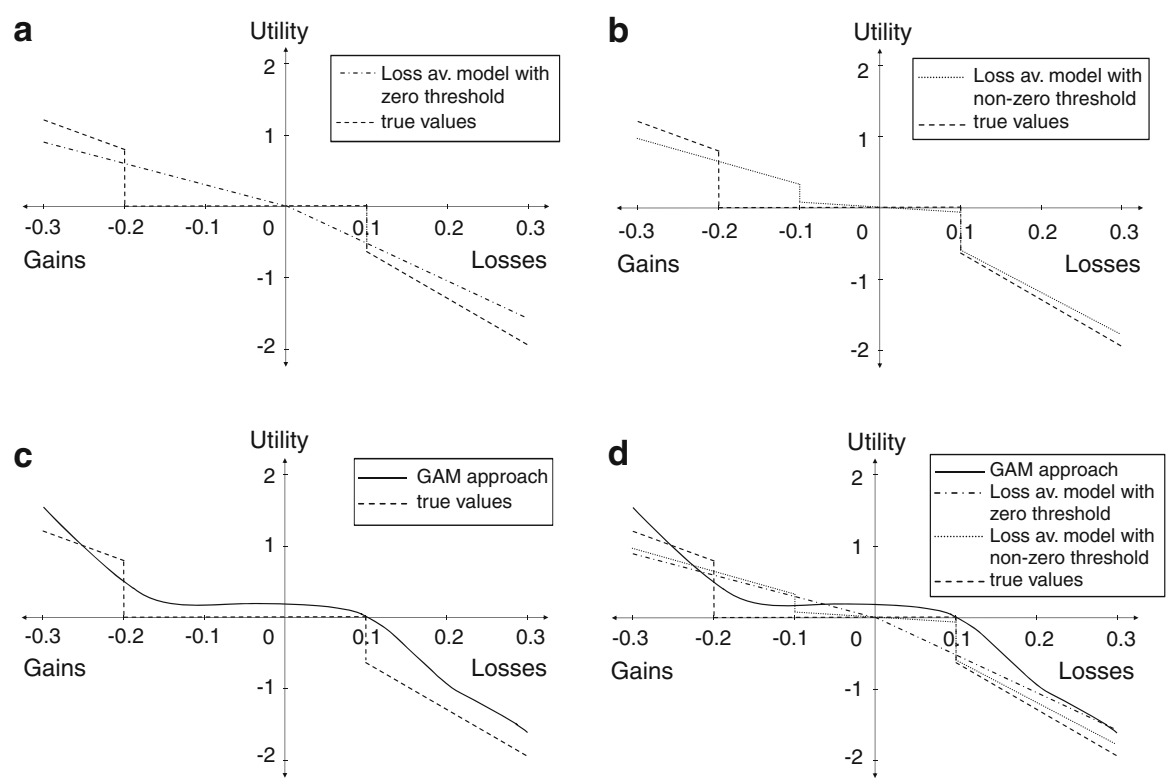

Fig. 3 Semi-parametric and parametric estimation for the price gap in the simulation study with data based on a loss aversion model with non-zero threshold. a The estimation for a model based on loss aversion with zero threshold. $\mathbf{b}$ The estimation for a model based on loss aversion with non-zero threshold. $\mathbf{c}$ The semi-parametric estimation. $\mathbf{d}$ A combination of $\mathbf{a}-\mathbf{c}$

use of a loss aversion model with non-zero threshold, non-parametric estimates can be applied to provide guidance on the width and even the symmetry (or lack thereof) of the indifference zone.

\subsection{Real data set}

The database consists of purchases of one product category in a micro test market. Confidentiality restrictions prevent us from revealing the brand and category names. Subject to these confidentiality constraints, we provide the following limited information about the data set. The data span 104 weeks and 7 brands of merchandise from a major retail store. The product category belongs to daily care products. The category is a typical fast moving consumer good, which is bought frequently by the consumers. All major brands in the category are included in our data set. During the specified time period, 876 households made 3,647 purchases. For calibration purposes, we use the data for the first 80 weeks aside. The remaining 24 weeks are used for validation. The data consisted of purchase information about the actual price paid and display and feature levels. From these data, we constructed the loyalty variable proposed by Guadagni and Little (1983). The literature suggests many different specifications for reference price concept (internal and external). In this paper, we use the reference price specification that leads to the best model fit as determined by the log-likelihood value. Based on this criterion, we present the results for the weighted mean of last 
prices as internal reference price and the actual price of last brand bought as external reference price.

In the weighted mean of last prices (e.g., Winer 1985), not all former prices have the same weight in the calculation, and these weights must be estimated, a task which increases the estimation complexity. This form of the internal reference price is similar to the loyalty measure introduced by Guadagni and Little (1983) and can be modeled as $I R P_{i n t}=\zeta I R P_{i n t-1}+(1-\zeta)\left[P_{i n t-1}-I R P_{i n t-1}\right]$.

The logic of the Guadagni and Little (1983) specification for $I R P_{i n t}$ is straightforward. The current internal reference price is a weighted linear (convex) combination of the previous period's internal reference price and its deviation from the previous period's actual price. The linear combination is called convex because the weights add up to one.

For the external reference price $\left(E R P_{n t}\right)$, the actual price of the last brand bought (e.g., Hardie et al. 1993) is used in cases when consumers cannot remember the last price paid, but can remember the last brand bought. It can be modeled as $E R P_{n t}=P_{i t}$ if $i$ was bought in $t-1$. Raman and Bass (2002) show that a rational expectations approach, operationalized through Box and Jenkins' ARIMA technology, also leads to the actual price of the last brand bought.

\subsubsection{Consideration of heterogeneity}

Ignoring consumer heterogeneity could lead to incorrect parameter estimates (e.g., Chang et al. 1999). ${ }^{4}$ To take consumer heterogeneity into account, we follow the lead taken by Mazumdar and Papatla (1995, 2000), Krishnamurthi and Raj (1991), and Krishnamurthi et al. (1992). These authors create a priori segments of loyal and non-loyal consumers. ${ }^{5}$ Assuming that loyal consumers are not very price sensitive, it follows that they use an external reference price. Non-loyal consumers are much more price-conscious and thus are able to use an internal reference price.

Following the lead of these authors, we account for heterogeneity of preference through the loyalty variable. Heterogeneity of response is captured by brand intercepts; structural heterogeneity is accounted for through segmentation of consumers.

In Table 5, we first look at the parametrically estimated results, which are given in columns 1-4. We estimate one approach based on a loss aversion model with zero threshold and one approach based on a loss aversion model with non-zero threshold. As described before, we estimate one part for non-loyal consumers using IRP and the other part for loyal consumers using ERP. Display and feature have the expected magnitudes and signs. The model based on a zero threshold effect shows different

\footnotetext{
4 For a brief presentation of different heterogeneity concepts, the reader is referred to DeSarbo et al. (1999).

5 We also used a latent class approach (Kamakura and Russell 1989; Jain et al. 1994) limited to two segments, but the results are close to the a priori segmentation. The results of the a priori segmentation can be interpreted more easily so only those results are used in the following. Another reason for not using the latent class approach is that in that method, group membership is only weakly related to secondary drivers data (DeSarbo et al. 1997). Erdem et al. (2001) explicitly demonstrated the problems that arise when using a latent class approach for a reference price model.
} 
Table 5 Estimation results for real data set incorporating heterogeneity

\begin{tabular}{|c|c|c|c|c|c|c|}
\hline & \multicolumn{2}{|c|}{$\begin{array}{l}\text { Loss aversion model } \\
\text { with zero threshold }\end{array}$} & \multicolumn{2}{|c|}{$\begin{array}{l}\text { Loss aversion model } \\
\text { with non-zero threshold }\end{array}$} & \multicolumn{2}{|c|}{ Semi-parametric approach } \\
\hline & IRP & ERP & IRP & ERP & IRP & ERP \\
\hline$P$ & -3.55 & n.a. & -2.04 & n. a. & -0.87 & n. a. \\
\hline DISPL & 0.69 & 0.58 & 0.67 & 0.58 & 0.61 & 0.57 \\
\hline FEAT & 1.27 & 1.01 & 1.24 & 1.06 & 1.10 & 0.98 \\
\hline Gain & 3.22 & 0.20 & 4.08 & 0.05 & & \\
\hline Mid & n. a. & n. a. & -14.39 & -5.22 & Plot & Plot \\
\hline Loss & -3.99 & -7.64 & -3.47 & -6.75 & & \\
\hline$L O Y$ & 2.55 & 7.34 & 2.72 & 8.41 & 2.50 & 7.51 \\
\hline$\delta$ & n.a. & & 0.1 & & Plot & \\
\hline$\ell$ & $-3,589$ & & $-3,581$ & & $-3,545$ & \\
\hline $\bar{\rho}^{2}$ & 0.317 & & 0.318 & & 0.324 & \\
\hline
\end{tabular}

Bolded values indicate significant parameter estimates

Table 6 Estimation results for real data set incorporating heterogeneity based on loss aversion model with non-zero threshold, with parametrically and non-parametrically produced $\delta$ values

\begin{tabular}{lllll}
\hline & $\begin{array}{l}\text { Loss aversion model with } \\
\text { non-zero threshold and } \delta \text { symmetric } \\
\text { (suboptimal) }\end{array}$ & $\begin{array}{l}\text { Loss aversion model with non-zero } \\
\text { threshold and } \delta \text { detected from } \\
\text { non-parametric estimation results } \\
\text { (optimal) }\end{array}$ \\
\cline { 2 - 3 } \cline { 5 - 5 } & IRP & ERP & IRP & ERP \\
\hline$P$ & $-\mathbf{2 . 0 4}$ & n. a. & $-\mathbf{1 . 8 0}$ & n. a. \\
DISPL & $\mathbf{0 . 6 7}$ & $\mathbf{0 . 5 8}$ & $\mathbf{0 . 6 4}$ & $\mathbf{0 . 6 0}$ \\
FEAT & $\mathbf{1 . 2 4}$ & $\mathbf{1 . 0 6}$ & $\mathbf{1 . 2 4}$ & $\mathbf{1 . 0 3}$ \\
Gain & $\mathbf{4 . 0 8}$ & 0.05 & $\mathbf{4 . 3 4}$ & 0.77 \\
Mid & $-\mathbf{1 4 . 3 9}$ & -5.22 & $-\mathbf{1 3 . 4 1}$ & 2.61 \\
Loss & $-\mathbf{3 . 4 7}$ & $-\mathbf{6 . 7 5}$ & $-\mathbf{1 . 9 4}$ & $-\mathbf{7 . 2 1}$ \\
LOY & $\mathbf{2 . 7 2}$ & $\mathbf{8 . 4 1}$ & $\mathbf{2 . 8 4}$ & $\mathbf{8 . 4 4}$ \\
$\delta$ & 0.1 & 0.1 & 0.14 & $0.1 / 0.04$ \\
$\ell$ & $-3,581$ & & $-3,575$ & \\
$\bar{\rho}^{2}$ & 0.318 & & 0.319 & \\
\hline
\end{tabular}

Bolded values indicate significant parameter estimates

parameter values for both consumer segments, particularly for all price components (e.g., price, loss, and gain) and for the loyalty variable.

The estimation results for the model based on a non-zero threshold effect are similar to those from the zero threshold model except that all price component values are larger. However, for loyal consumers, the gain and the mid range components are not significant. The optimal width of the indifference zone is 0.1 . The width is found by a grid search, as explained in Sect. 3. 

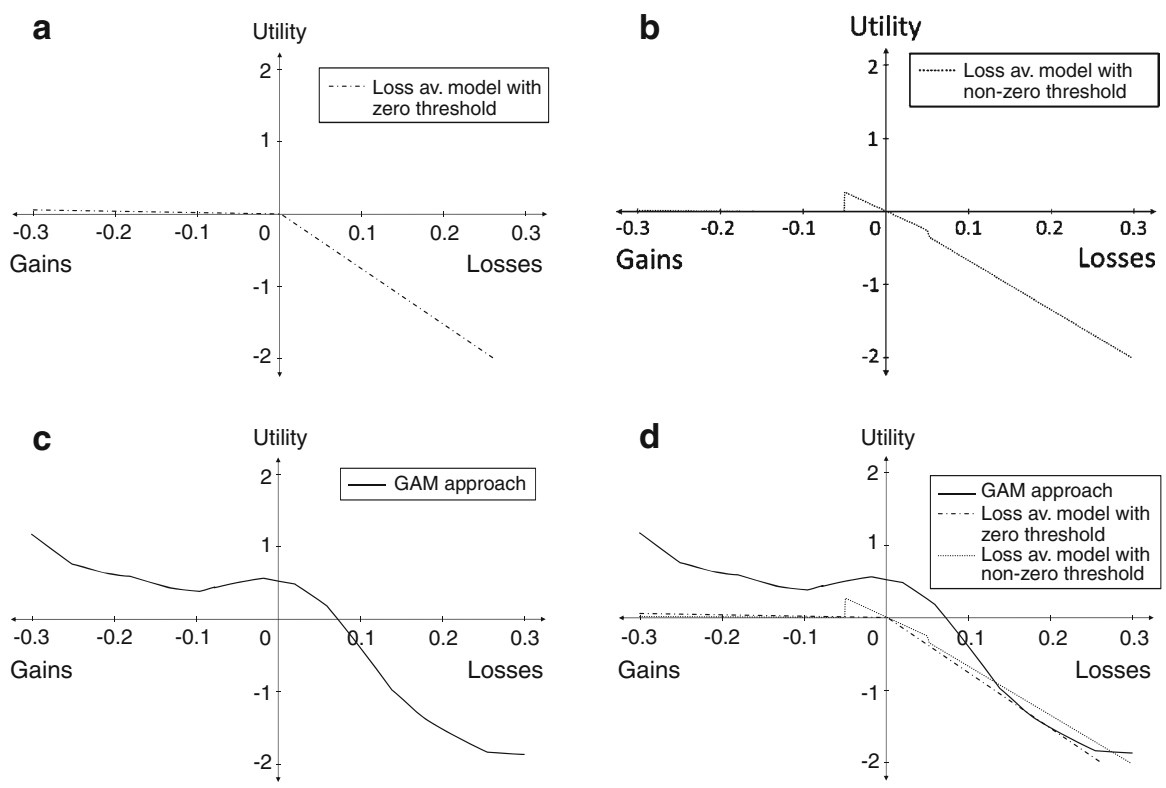

Fig. 4 Semi-parametric and parametric estimation for the price gap in the real data set for loyal consumers incorporating heterogeneity. a The estimation for a model based on loss aversion with zero threshold. b The estimation for a model based on loss aversion with non-zero threshold. c The semi-parametric estimation. d A combination of $\mathbf{a}-\mathbf{c}$

The most interesting discovery of the semi-parametric approach is that loyal and non-loyal customers are described by entirely different perceptual processes. Loyal customers are clearly following a non-zero threshold model (see Fig. 4) whereas nonloyal customers are described best by a zero threshold model (see Fig. 5). This is intuitively sensible since we would expect that loyal customers would tolerate small price changes whereas non-loyal customers would react to even small price changes. The parameter estimates for the variables not related to price (display, feature, and loyalty) are very close to the results from the parametric approaches.

As pointed out previously, the non-parametric estimates are used in two ways. First, we use it to detect which theory best represents the underlying data set. Second, if the first step shows non-zero threshold to be the best description of the data, we additionally use the non-parametric estimates to obtain the optimal $\delta_{\text {Gain }}$ and $\delta_{\text {Loss }}$ for the width of the zone of indifference.

Figures 4 and 5 show that, for the segment of non-loyal consumers using an internal reference price, the zone of indifference is a symmetric interval with a width of $\delta_{\text {Gain }}=$ $\delta_{\text {Loss }}=0.07$, respectively, $\delta_{\text {Gain }}+\delta_{\text {Loss }}=0.14$. Loyal consumers using an external reference price have an asymmetric zone, with a width of $\delta_{\text {Gain }}=0.1$ on the gain side and $\delta_{\text {Loss }}=0.04$ on the loss side. With these new values for $\delta_{\text {Gain }}$ and $\delta_{\text {Loss }}$, we reestimate our parametric model incorporating heterogeneity by separating consumers into loyal and non-loyal segments.

Table 6 compares the estimation based on a loss aversion model with non-zero threshold with symmetric $\delta$ (as already presented in Table 5) and, due to the parametric 
a

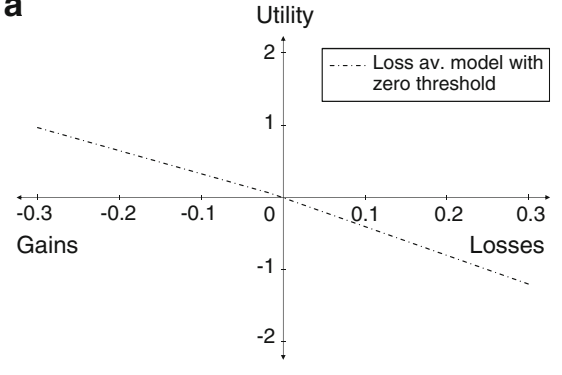

C

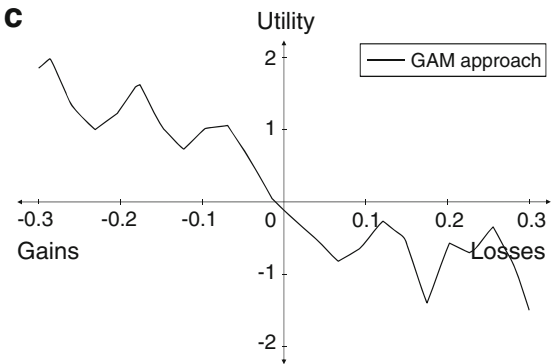

b

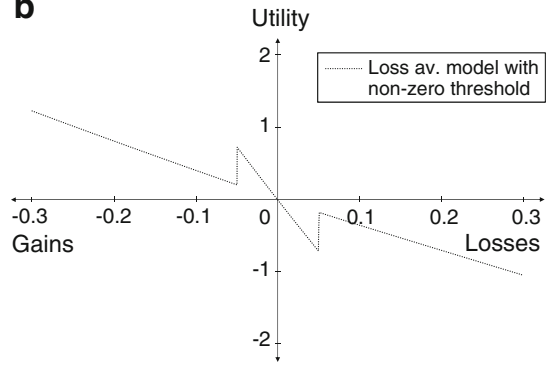

d

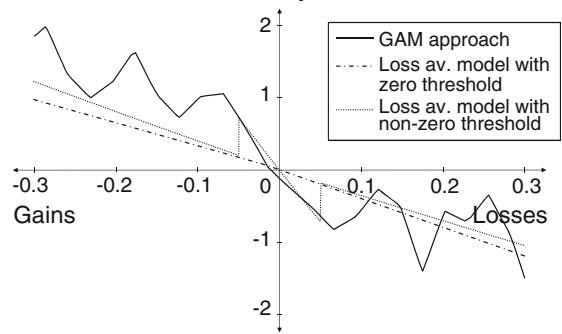

Fig. 5 Semi-parametric and parametric estimation for the price gap in the real data set for non-loyal consumers incorporating heterogeneity. a The estimation for a model based on loss aversion with zero threshold. b The estimation for a model based on loss aversion with non-zero threshold. $\mathbf{c}$ The semiparametric estimation. $\mathbf{d}$ A combination of $\mathbf{a}-\mathbf{c}$

model restriction, "suboptimal" $\delta_{\text {Gain }}$ and $\delta_{\text {Loss }}$ with the model using $\delta_{\text {Gain }}$ and $\delta_{\text {Loss }}$ values extracted from Figs. 4 and 5. Due to a slightly larger zone of indifference in the model with $\delta_{\text {Gain }}$ and $\delta_{\text {Loss }}$ from the non-parametric estimation, all price-related values change slightly, the largest changes being to the gain and mid range components in the external reference price model. Most important is the significant improvement in model fit, which is also reflected in the predictive accuracy for the holdout samples.

\section{Managerial implications}

It has long been known that the reference price used by consumers is one of the key determinants of their purchase behavior. Our semi-parametric method identifies whether zero or non-zero thresholds best describe consumer behavior based on the observed data. For the most part, published studies of reference price effects have assumed a model with zero threshold so that every deviation of the price from the reference price, no matter how small, has a substantial influence on consumer behavior-a view that is clearly strongly driven by prospect theory. However, we find that both zero and non-zero thresholds are possible - a finding that is managerially significant because of the dramatically different implications of zero and non-zero thresholds for managing promotions. 
We find that whether zero or non-zero thresholds best describe consumer purchase behavior is contingent upon loyalty characteristics. Loyal and non-loyal consumers respond differently to price promotions. Loyal consumers behave according to a nonzero threshold model and furthermore, the indifference zone is asymmetric, being longer on the gains side and shorter on the loss side. The promotional implications of this finding are noteworthy-for one thing, it suggests that small promotions will not be worthwhile to the firm because they will result in a loss of revenue since consumers will not respond to price reductions within the zone of indifference. Unless the promotion is big enough to exceed the threshold, it will not have a negligible effect. The behavior of non-loyal consumers is consistent with a zero threshold model and this finding has significantly different implications for promotions compared to the non-zero threshold model. For the non-loyal segment of consumers, we found that response is very steep around the zero region of the gap between price and reference price. Clearly, what this finding suggests is that non-loyal customers will respond even to promotions of small magnitude. Thus a large promotional reduction may not only be unnecessary - it could be seriously suboptimal from a profitability perspective.

To sum it up, the implications of zero and non-zero thresholds are not just of theoretical importance-rather they have important managerial implications. Thus, managers should first find out which of these situations best describes their customer segments and then tailor their price promotions in the segment accordingly. Our semiparametric method facilitates distinguishing between the zero and non-zero threshold models.

\section{Conclusions}

We developed a semi-parametric technique, which when used in conjunction with traditional parametric methods in a two-stage fashion, enables distinguishing between two important competing theories of consumer behavior in the first stage and estimating the relevant model of price response in the second stage. By doing so, managers can structure their price promotions more effectively by basing them on the correct model of price response. Our simulations showed very clearly that the wrong model often gives significant results and a good fit to the data, thereby misleading researchers into exactly the wrong conclusions about consumer response and consequently, designing promotions sub-optimally. Our contribution is to offer a methodology that can identify the correct model and estimate consumer response to price more efficiently compared to existing techniques.

We note the following limitations of our research, which could serve as motivations for future research. Out of the extensive literature on reference prices, we considered only two models of the reference price effect. Our rationale for doing so was that these two are the predominantly used models in the literature. Another limitation is that our estimation method works in two stages - it would be desirable to have an integrated estimation framework that combines these stages seamlessly. A potentially interesting area for future research would be to disentangle different processes that underlie reference price formation from the consumer's point of view so that we can understand the different processes that may explain loss versus gain asymmetry 
(prospect theory offers one explanation). We see these as promising areas for future work and hope that our paper encourages other researchers to pursue these directions.

Open Access This article is distributed under the terms of the Creative Commons Attribution License which permits any use, distribution, and reproduction in any medium, provided the original author(s) and the source are credited.

\section{References}

Biswas A, Wilson EJ, Licata JW (1993) Reference pricing studies in marketing: a synthesis of research results. J Bus Res 27(3):239-256

Briesch RA, Krishnamurthi L, Mazumdar T, Raj SP (1997) A comparative analysis of reference price models. J Consum Res 24(2):202-214

Chang K, Siddarth S, Weinberg CB (1999) The impact of heterogeneity in purchase timing and price responsiveness on estimating of sticker shock effects. Market Sci 18(2):178-192

DeSarbo WS, Ansari A, Chintagunta P, Himmelberg C, Jedidi K, Johnson R, Kamakura W, Lenk P, Srinivasan K, Wedel M (1997) Representing heterogeneity in consumer response models. Market Lett 8(3):335-348

DeSarbo WS, Kim Y, Fong D (1999) A Bayesian multidimensional scaling procedure for the spatial analysis of revealed choice data. J Econ 89(1-2):79-108

Erdem T, Mayhew G, Sun B (2001) Understanding reference-price shoppers: a within- and cross-category analysis. J Market Res 38(4):445-457

Estelami H, Lehmann DR, Holden AC (2001) Macro-economic determinants of consumer price knowledge: a meta-analysis of four decades of research. Int J Res Market 18(4):341-355

Friedman JH, Stuetzle W (1981) Projection pursuit regression. J Am Stat Assoc 76(376):817-823

Gijsbrechts E (1993) Prices and pricing research in consumer marketing: some recent developments. Int J Res Market 10(2):115-151

Guadagni PM, Little JDC (1983) A logit model of brand choice calibrated on scanner data. Market Sci 2(3):203-238

Han S, Gupta S, Lehmann DR (2001) Consumer price sensitivity and price thresholds. J Retail 77(4):435456

Hardie BGS, Johnson EC, Fader PS (1993) Modeling loss aversion and reference dependence effects on brand choice. Market Sci 12(4):378-394

Hastie T, Tibshirani R (1990) Generalized Additive Models. Chapman \& Hall, London

Jain DC, Vilcassim NJ, Chintagunta PK (1994) A random-coefficients logit brand-choice model applied to panel data. J Bus Econ Stat 12(3):317-328

Janiszewski C, Lichtenstein DR (1999) A range theory account of price perception. J Consum Res 25:353368

Kahneman D, Tversky A (1979) Prospect theory: an analysis of decision under risk. Econometrica 47(2):263-291

Kalyanaram G, Little JDC (1994) An empirical analysis of latitude of price acceptance in consumer package goods. J Consum Res 21(4):408-418

Kalyanaram G, Winer RS (1995) Empirical generalization from reference price research. Market Sci 14(3):G161-169

Kamakura WA, Russell GJ (1989) A probabilistic choice model for market segmentation and elasticity structure. J Market Res 26(4):379-390

Krishnamurthi L, Mazumdar T, Raj SP (1992) Asymmetric response to price in consumer brand choice and purchase quantity decisions. J Consum Res 19(3):387-400

Krishnamurthi L, Raj SP (1991) An empirical analysis of the relationship between brand loyalty and consumer price elasticity. Market Sci 10(2):172-183

Linton OB, Nielsen JP (1995) A kernel method of estimating structured nonparametric regression models. Biometrika 84(2):469-473

Mazumdar T, Papatla P (1995) Loyalty differences in the use of internal and external reference prices. Market Lett 6(2):111-122 
Mazumdar T, Papatla P (2000) An investigation of reference price segments. J Market Res 37(2):246-258

Mazumdar T, Raj SR, Sinha I (2005) Reference price research: review and propositions. J Market 69(4):84102

McCullagh P, Nelder JA (1989) Generalized linear models. Chapman and Hall, London

McFadden D (1974) Conditional logit analysis of qualitative choice behavior. In: Zarembka P (ed) Frontiers in econometrics. Academic Press, New York, pp 105-142

Niedrich RW, Sharma S, Wedell DH (2001) Reference price and price perceptions: a comparison of alternative models. J Consum Res 28:339-354

Pauwels KH, Franses PH, Srinivasan S (2003) Reference-based transitions in short-run price elasticity. ERIM report series, reference no. ERS-2003-095-MKT

Rajendran KN, Tellis GJ (1994) Contextual and temporal components of reference price. J Market 58(1): 22-34

Raman K, Bass FM (2002) A general test of reference price theory in the presence of threshold effects. Tijdschrift voor Economie en Management (J Econ Manag) 47(2):205-226

Sherif M, Hovland CI (1961) Social judgment. Yale University Press, New Haven

Steiner WJ, Brezger A, Belitz C (2007) Flexible estimation of price response function using retail scanner data. J Retail Consum Serv 14(6):383-393

Tversky A, Kahneman D (1981) The framing of decisions and the psychology of choice. Science 211(4481):453-458

van Heerde HJ, Leeflang PSH, Wittink DR (2001) Semi parametric analysis to estimate the deal effect curve. J Market Res 38:197-215

Winer RS (1985) A price vector model of demand for consumer durables: preliminary developments. Market Sci 4(1):74-90

Winer RS (1986) A reference price model of brand choice for frequently purchased products. J Consum Res 13(2):250-256

Winer RS (1988) Behavioral perspective on pricing: buyers' subjective perceptions of price revisited. In: Devinney TM (ed) Issues pricing. Lexington Books, Lexington, pp 35-57

Wricke M, Herrmann A, Huber F (2000) Behavioral pricing. WiSt 29(12):692-697 